\title{
The anti-atherosclerotic effects of puerarin on induced-atherosclerosis in rabbits
}

\author{
Lidao Bao ${ }^{\mathrm{a}}$, Ying Zhang ${ }^{\mathrm{b}}$, Guomin Wei ${ }^{\mathrm{b}}$, Yi Wang ${ }^{\mathrm{a}}$, Ruilian $\mathrm{Ma}^{\mathrm{a}}$, Rui Cheng ${ }^{\mathrm{a}}$, Xianhua Rena ${ }^{\mathrm{a}}$, Agula Bc
}

\begin{abstract}
Background. Cardiovascular disease is a major health issue worldwide, which has been well treated by the extracts of traditional herbs. Radix Puerariae, the dried root of the leguminous plant Pueraria lobata (Willd.) Ohwi, is a delicious vegetable in some southern provinces of China. Puerarin has also been widely used to treat human diseases, but few controlled studies are available.
\end{abstract}

Aim. To determine the anti-atherosclerotic effects of puerarin on fat diet-induced atherosclerosis (AS) in rabbits.

Methods. An AS model was established by feeding 60 rabbits a high-fat diet and randomly dividing them into 6 groups: (1) normal control, (2) a model group (3) the statin, simvastatin and groups (4), (5) and (6) received 3 different amounts of puerarin. The fasting sera of all animals were collected before and after 90 days treatment to determine the levels of total cholesterol (TC), triglyceride (TG), low density lipoprotein-cholesterol (LDL-C) and high density lipoproteincholesterol (HDL-C). The aortas were pathomorphologically examined. PCNA and PDGF-A protein levels were assessed by Western blot.

Results. On the 90th day, the levels of TC, TG and LDL-C were significantly lower in high- and middle-dose puerarin groups and simvastatin group than in the model group $(P<0.05)$, and the HDL-C level was higher. The percentages of plaque area to the total aortic area differed significantly for high- and middle-dose puerarin groups, simvastatin group and model group $(P<0.05)$. Whole blood viscosity increased in the high-fat diet groups, while those in the treatment groups (except for the low-dose puerarin group) were significantly lower than those in the model group $(P<0.05)$. PCNA and PDGF-A protein expression levels of rabbit aorta were low in the normal group. Protein expression levels in the groups fed the high-fat diet were significantly increased $(P<0.05)$, but those in the high-dose puerarin group and simvastatin group were significantly decreased compared with the model group $(P<0.05)$.

Conclusions. Puerarin inhibits the formation and development of AS plaque and suppresses the migration and reproduction of vascular smooth muscle cells by decreasing PCNA and PDGF-A expressions in the rabbit. This is encouraging in terms of cardiovascular disease prevention/treatment.

Key words: puerarin, rabbit, atherosclerosis, proliferation cell nuclear antigen, platelet-derived growth factor

Received: July 7, 2013; Accepted: December 19, 2013; Available online: January 27, 2014

http://dx.doi.org/10.5507/bp.2013.096

${ }^{a}$ Department of Pharmacy, Affiliated Hospital of Inner Mongolia Medical University, Hohhot 010059, P. R. China

${ }^{b}$ Department of Respiratory Medicine, Binzhou People's Hospital, Binzhou 256610, P. R. China

'College of Traditional Mongolia Medicine, Inner Mongolia Medical University, Hohhot 010110, P. R. China

Corresponding author: Lidao Bao, e-mail: lidao_bao@163.com

\section{INTRODUCTION}

Atherosclerosis (AS) is a cause of cardiovascular and cerebrovascular diseases (CCVD) ${ }^{1}$, and hence to prevent and reduce AS is of paramount importance. The migration and proliferation of vascular smooth muscle cells (VSMC) are key steps in the process of AS formation. Proliferating cell nuclear antigen (PCNA), which is a nuclear protein, is a reliable indicator of cell proliferation status $^{2}$. Its content changes in the cell cycle are consistent with those in DNA synthesis. In the pathological changes of AS, the nuclear factor kB (NF-kB) activates and induces expression of platelet-derived growth factor(PDGF) while PDGF is an important element in inducing SMC proliferation and migration from arterial media to intima ${ }^{3}$.

Radix Puerariae, as the dried root of the leguminous plant Pueraria lobata (Willd.) Ohwi, is an edible vegetable in some southern provinces in China and is commonly used in stewing soup for its sweet, cool and delicious taste.
The main ingredient of puerarin is starch, and in addition, it contains about $12 \%$ of flavonoid compounds, including more than 10 active ingredients, such as daidzein, daidzin, puerarin and puerarin-7-xyloside, etc.; it also contains daucosterol, amino acid and coumarin, which can be used as medicine ${ }^{4,5}$. Its functions are to enhance the regenerative capacity of liver cells, restore normal hepatic function, promote bile secretion, prevent the accumulation of fat in liver, enhance metabolism, improve the status of myocardial ischemia and cerebral arteriosclerosis and strengthen the autoimmune function of hepatobiliary cells $\mathrm{s}^{6,7}$.

Puerarin plays a positive role in improving immunity, enhancing myocardial contractility, dilating blood vessels, lowering blood pressure, boosting microcirculation, as well as myocardial cell protection and anti-platelet aggregation $^{8-11}$. It can reduce the immune impaired liver tissue, effectively reverse chemically-induced liver fibrosis ${ }^{12}$, and also has a protective effect on nephritic and renal failure; it promotes the lymphocyte transformation rate in 
healthy subjects and cancer patients, enhances the role of natural hormones, and exerts significant stimulatory and inductive effects on the interference system ${ }^{13-15}$. This study aims to identify the anti-atherosclerotic effects of puerarin through the observation of puerarin on experimental rabbits with AS, and examine its influence on the PCNA and PDGF protein expressions of aortas using the atherosclerotic rabbit model.

\section{MATERIALS AND METHODS}

\section{Drugs and reagents}

Puerarin was purchased from Xi'an Sobeo Biotech Co., Ltd. (batch No. 20110125), ground with 0.5\% sodium carboxymethyl cellulose and prepared into a suspension. Simvastatin was bought from Shandong Luoxin Pharmacy Stock Co.,Ltd. (batch No. 100513) and dissolved in distilled water. Cholesterol was obtained from Beijing Aipoo HuaMei Biotechnology Co., Ltd. (batch No. 20101215). Total cholesterol (TC), triglyceride (TG), high density lipoprotein-cholesterol (HDL-C) and low density lipoprotein-cholesterol (LDL-C) kits were bought from Zhongsheng Beikong Biotechnology Co., Ltd. RNA extraction and reverse transcriptase polymerase chain reaction (RT-PCR) kits were purchased from TaKaRa (Dalian). Protein extraction Trizol lysate was provided by Beijing Biosynthesis Biotechnology Co., Ltd. Rabbit anti-PCNA and rabbit anti-PDGF-A antibodies were obtained from Santa Cruz Biotechnology, inc.

\section{Animals}

Healthy Japanese male white rabbits weighing $(2.0 \pm 0.2) \mathrm{kg}$ were provided by the Department of Laboratory Animal Science, Health Science Center, Peking University (license No. SCXK (Beijing) 2005-0002). Animals were housed and maintained at the Inner Mongolia Medical University at $(18 \pm 2)^{\circ} \mathrm{C}$ and relative humidity of $(40 \pm 15) \%$, and all experiments were treated in compliance with protocols and policies approved by the Animal Care and Use Committee of Inner Mongolia Medical University.

\section{Instruments}

We used the following instruments : PRONTO-E Automatic Biochemical Analyzer (PRONTO, Italy), Z216MK micro-volume high-speed refrigerated centrifuge (HERMLE, Germany), MOTIC AE20 biomicroscope (Motic China Group Co., Ltd.), South990 Auto Hematology Analyzer (Chongqing Nanfang Numerical Control Equipment Co., Ltd.), DU730 protein-nucleic acid spectrophotometer (Beckman, USA), Kodak image station 2000MM imaging system (KODAK, USA).

\section{Animal groups and treatment}

Sixty rabbits were fed separately a standard diet in an experimental environment for three weeks. The 60 rabbits were then randomly divided into 6 groups, with 10 rabbits in each group. The normal control group (1) was fed the standard diet and the other five groups with a high-fat diet $(1.5 \%$ cholesterol, $5 \%$ lard, $10 \%$ egg yolk powder and $83.5 \%$ basal feed). The normal group and model group (2) were administered distilled water by gastric gavage. The positive control group (3) was given $5 \mathrm{mg} \cdot \mathrm{kg}^{-1} \cdot \mathrm{d}^{-1}$ simvastatin, and the high- (4), middle- (5) and low-dose (6) puerarin groups given puerarin of $0.4 \mathrm{~g}, 0.2 \mathrm{~g}, 0.1 \mathrm{~g} . \mathrm{kg}^{-1} \cdot \mathrm{d}^{-1}$ respectively through intragastric administration, with a volume of $8 \mathrm{~mL}$ per $\mathrm{kg}$ of body weight, once a day for 90 consecutive days. The rabbits were ear-intravenously anesthetized with $2 \mathrm{~mL} / \mathrm{kg} \mathrm{10 \%}$ chloral hydrate that was dissolved prior to use. The blood samples were then collected from heart after the end of the experiment and then all the rabbits were killed. The aorta was removed as soon as possible to peel off the adipose tissue on the aortic wall, cut it along the posterior surface in a longitudinal direction, spread and we observed the plaque with the naked eye. Subsequently, tissue $1 \mathrm{~cm}$ under the aortic arch was collected and fixed with 10\% neutral formaldehyde solution, and the rest was fixed with $15 \%$ formaldehyde.

\section{Observation and detection indices}

Under normal conditions, the basic status of the rabbits was observed daily including emotional state, behavior activity, hair color, feeding, water drinking and feces, etc. The body weight was measured once every 30 days to calculate the average weight of each group. The blood was collected from ear marginal veins $12 \mathrm{~h}$ after fasting one day before the experiment and on the $30^{\text {th }}$ and $60^{\text {th }}$ day after the start of the test respectively. Blood was sampled from the heart on the $90^{\text {th }}$ day to separate blood serum, and then the levels of TC, TG, LDL-C and HDL-C were measured respectively on the automatic biochemical analyzer for statistical analysis. The atherogenic index $(\mathrm{AI})=(\mathrm{TC}-\mathrm{HDL}-\mathrm{C}) / \mathrm{HDL}-\mathrm{C}$ was calculated. The whole blood hemorheology analysis was made timely after the hemorheology detection on heart blood samples. For the pathomorphological examination, samples were obtained from the aortic specimen fixed in $10 \%$ neutral formaldehyde solution by general method, conducted with routine paraffin embedding, slicing and HE staining, and elastic fiber staining was also performed on aortic tissues to observe and photograph by light microscopy. The MOTIC AE20 microscope and high-definition color medical image analysis system (HMIAS-2000) were adopted to conduct pathological and quantitative analysis with random graphing, measure the intimal thickness (I) and medial thickness (M) of aortic slices after HE staining, and to calculate the $\mathrm{I} / \mathrm{M}$.

\section{Western blot detection}

The aortas in the freezing tube of high-dose puerarin were ground in freezing condition for cell lysis with Trizol and protein extraction. Spectrophotometer was used to measure the total protein concentration for protein quantification. The trough for gel pouring was assembled, and SDS-PAGE gel and protein samples for electrophoresis were prepared. The electrophoretic condition was at $120 \mathrm{~V}$ for $2.5 \mathrm{~h}$. An electroporation instrument was used 
to transfer the protein onto the PVDF membrane at a constant voltage $15 \mathrm{~V}$ for $15 \mathrm{~min}$. Then the PVDF membrane was transferred to a large petri dish and a blocking solution was added for blocking on a shaking table at ambient temperature overnight. The PVDF membrane was made to react with a variety of primary antibodies diluted by 1:200 at room temperature for $4 \mathrm{~h}$, and then react with secondary antibodies diluted by $1: 1000$ at room temperature for $1.5 \mathrm{~h}$. The ECL chemiluminescence detection was conducted as follows: equal amounts of solution A and B were mixed in the ECL to fully contact with the PVDF membrane for 1min, to drain redundant ECL reagent on the membrane, then place the PVDF membrane exposed under the Kodak image Station 2000MM imaging system to obtain the target strip images. Then the ImageTool3.0 analysis software was adopted to detect the expression levels of PCNA and PDGF-A and obtain images. The image processing software ImageTool3.0 was used to measure the gray value, obtain the relative value compared to the $\beta$-actin internal control, and make comparison among the mean values of the results of three repeated experiments.

\section{Statistical analysis}

All data were analyzed by SPSS 17.0 and subjected to normality tests by 1 -SampleK-S, and those followed normal distribution were compared. The eligible data were analyzed by One-Way ANOVA LSD program or Tambane's T2 method. The data were expressed as mean \pm standard deviation $(\mathrm{x} \pm \mathrm{SD})$, and the means were compared by $t$ tests. The data that did not conform to a normal distribution with variance heterogeneity were compared by Wilcoxon rank sum test, and expressed as means \pm interquartile range $(\mathrm{M} \pm \mathrm{Q}) . P<0.05$ was considered statistically significant.

Table 1. Effects of puerarin on serum lipids $(\mathrm{mmol} / \mathrm{L}, \mathrm{x} \pm \mathrm{s})$.

\begin{tabular}{|c|c|c|c|c|c|c|c|}
\hline Item & Group & $\mathrm{n}$ & Dose $(\mathrm{mg} / \mathrm{kg})$ & 0 day & $30^{\text {th }}$ day & $60^{\text {th }}$ day & $90^{\text {th }}$ day \\
\hline \multirow[t]{6}{*}{$\mathrm{TC}$} & 1 & 10 & & $1.42 \pm 0.22$ & $1.76 \pm 0.35^{*}$ & $2.33 \pm 0.55^{*}$ & $2.42 \pm 0.58^{*}$ \\
\hline & 2 & 10 & & $1.35 \pm 0.30$ & $28.24 \pm 3.36$ & $34.71 \pm 2.88$ & $43.62 \pm 5.10$ \\
\hline & 3 & 10 & 5 & $1.33 \pm 0.24$ & $21.29 \pm 3.02 *$ & $29.85 \pm 1.45 *$ & $34.69 \pm 3.29 *$ \\
\hline & 4 & 10 & 400 & $1.31 \pm 0.14$ & $23.98 \pm 3.01 *$ & $30.63 \pm 3.96^{*}$ & $37.24 \pm 4.11$ * \\
\hline & 5 & 10 & 200 & $1.32 \pm 0.22$ & $24.75 \pm 3.56^{*}$ & $32.25 \pm 4.66^{*}$ & $39.62 \pm 5.02 *$ \\
\hline & 6 & 10 & 100 & $1.32 \pm 0.17$ & $25.77 \pm 4.24$ & $34.26 \pm 2.71$ & $40.07 \pm 4.29$ \\
\hline \multirow[t]{6}{*}{ TG } & 1 & 10 & & $1.28 \pm 0.26$ & $1.22 \pm 0.20$ * & $1.30 \pm 0.24 *$ & $1.42 \pm 0.25^{*}$ \\
\hline & 2 & 10 & & $1.24 \pm 0.17$ & $3.52 \pm 0.41$ & $3.69 \pm 0.83$ & $3.49 \pm 0.51$ \\
\hline & 3 & 10 & 5 & $1.30 \pm 0.25$ & $3.12 \pm 0.24 *$ & $3.20 \pm 0.34^{*}$ & $2.82 \pm 0.53^{*}$ \\
\hline & 4 & 10 & 400 & $1.27 \pm 0.16$ & $3.13 \pm 0.40^{*}$ & $3.21 \pm 0.45^{*}$ & $2.63 \pm 0.28 *$ \\
\hline & 5 & 10 & 200 & $1.24 \pm 0.28$ & $3.43 \pm 0.29$ & $3.39 \pm 0.51$ & $3.34 \pm 0.47^{*}$ \\
\hline & 6 & 10 & 100 & $1.31 \pm 0.24$ & $3.48 \pm 0.36$ & $3.77 \pm 0.47$ & $3.66 \pm 0.37$ \\
\hline \multirow[t]{6}{*}{ LDL-C } & 1 & 10 & & $1.05 \pm 0.15$ & $1.07 \pm 0.16^{*}$ & $1.05 \pm 0.16^{*}$ & $1.33 \pm 0.14 *$ \\
\hline & 2 & 10 & & $1.03 \pm 0.09$ & $11.38 \pm 1.79$ & $11.65 \pm 1.74$ & $13.69 \pm 1.52$ \\
\hline & 3 & 10 & 5 & $1.02 \pm 0.15$ & $6.24 \pm 2.02 *$ & $8.32 \pm 2.01 *$ & $11.26 \pm 2.23 *$ \\
\hline & 4 & 10 & 400 & $1.05 \pm 0.22$ & $6.15 \pm 2.11^{*}$ & $8.01 \pm 1.77^{*}$ & $10.76 \pm 2.41 *$ \\
\hline & 5 & 10 & 200 & $1.07 \pm 0.08$ & $7.99 \pm 2.17 *$ & $11.26 \pm 2.33 *$ & $11.79 \pm 2.31$ * \\
\hline & 6 & 10 & 100 & $1.11 \pm 0.13$ & $12.02 \pm 2.25$ & $12.25 \pm 2.41$ & $13.05 \pm 2.68$ \\
\hline \multirow[t]{6}{*}{ HDL-C } & 1 & 10 & & $0.46 \pm 0.16$ & $0.48 \pm 0.33$ & $0.41 \pm 0.25$ & $0.47 \pm 0.12$ \\
\hline & 2 & 10 & & $0.40 \pm 0.12$ & $0.72 \pm 0.22$ & $0.98 \pm 0.25$ & $0.78 \pm 0.30$ \\
\hline & 3 & 10 & 5 & $0.41 \pm 0.15$ & $0.65 \pm 0.25$ & $1.04 \pm 0.45$ & $1.18 \pm 0.25$ \\
\hline & 4 & 10 & 400 & $0.47 \pm 0.22$ & $0.77 \pm 0.18$ & $1.11 \pm 0.39$ & $1.23 \pm 0.19 *$ \\
\hline & 5 & 10 & 200 & $0.40 \pm 0.17$ & $1.30 \pm 0.44$ & $0.98 \pm 0.33$ & $0.95 \pm 0.12$ \\
\hline & 6 & 10 & 100 & $0.39 \pm 0.23$ & $1.33 \pm 0.42$ & $0.93 \pm 0.36$ & $0.91 \pm 0.22$ \\
\hline \multirow[t]{6}{*}{ AI } & 1 & 10 & & $3.86 \pm 2.22$ & $2.83 \pm 1.01$ & $6.53 \pm 5.11$ & $8.99 \pm 3.64$ \\
\hline & 2 & 10 & & $4.14 \pm 1.86$ & $23.56 \pm 3.55^{*}$ & $36.88 \pm 8.54^{*}$ & $60.24 \pm 13.65$ * \\
\hline & 3 & 10 & 5 & $2.71 \pm 1.74$ & $19.14 \pm 3.46^{*}$ & $27.74 \pm 7.01 *$ & $29.62 \pm 8.02 *$ \\
\hline & 4 & 10 & 400 & $4.22 \pm 2.52$ & $18.52 \pm 4.16^{*}$ & $26.99 \pm 6.35^{*}$ & $32.68 \pm 10.21$ * \\
\hline & 5 & 10 & 200 & $3.87 \pm 1.75$ & $20.22 \pm 4.68^{*}$ & $29.22 \pm 5.74^{*}$ & $33.49 \pm 5.65^{*}$ \\
\hline & 6 & 10 & 100 & $3.33 \pm 0.91$ & $23.66 \pm 4.15$ & $34.20 \pm 10.15$ & $46.85 \pm 8.06$ \\
\hline
\end{tabular}

Compared with the model group (2), ${ }^{*} P<0.05$. 
Table 2. Effects of puerarin on blood rheology $(\mathrm{M} \pm \mathrm{Q})$.

\begin{tabular}{cccccccc}
\hline Group & $\begin{array}{c}\text { Dose } \\
(\mathrm{mg} / \mathrm{kg})\end{array}$ & \multicolumn{2}{c}{ Whole blood viscosity $(\mathrm{np} / \mathrm{mPa} \cdot \mathrm{S})$} & \multicolumn{2}{c}{$\begin{array}{c}\text { Plasma } \\
\text { viscosity } \\
(\mathrm{mPa} \cdot \mathrm{S})\end{array}$} & $\begin{array}{c}\text { HCT } \\
(\%)\end{array}$ & $\begin{array}{c}\text { Erythrocyte } \\
\text { electrophoresis } \\
\text { time }(\mathrm{s})\end{array}$ \\
\hline 1 & & $3.69 \pm 0.62^{*}$ & $4.52 \pm 0.75^{*}$ & $11.87 \pm 2.23^{*}$ & $1.32 \pm 0.23^{*}$ & $0.44 \pm 0.25^{*}$ & $14.68 \pm 3.22^{*}$ \\
2 & & $29.72 \pm 23.25$ & $34.26 \pm 27.15$ & $46.52 \pm 22.17$ & $2.14 \pm 0.92$ & $0.52 \pm 0.15$ & $50.21 \pm 16.78$ \\
3 & 5 & $22.56 \pm 7.35$ & $23.15 \pm 8.52$ & $36.47 \pm 5.81$ & $2.44 \pm 0.15$ & $0.53 \pm 0.14$ & $33.26 \pm 17.44$ \\
4 & 400 & $14.22 \pm 1.85^{*}$ & $16.05 \pm 2.56^{*}$ & $24.16 \pm 3.10^{*}$ & $1.36 \pm 0.41^{*}$ & $0.41 \pm 0.11$ & $18.21 \pm 10.32$ \\
5 & 200 & $16.54 \pm 3.98^{*}$ & $17.44 \pm 2.14^{*}$ & $24.01 \pm 2.56^{*}$ & $1.82 \pm 0.82^{*}$ & $0.43 \pm 0.14$ & $19.54 \pm 5.48$ \\
6 & 100 & $22.05 \pm 10.03$ & $25.33 \pm 6.25$ & $28.96 \pm 14.26$ & $1.98 \pm 0.47$ & $0.46 \pm 0.19$ & $21.67 \pm 5.26$ \\
\hline
\end{tabular}

Compared with the model group (2), ${ }^{*} P<0.05$.

\section{RESULTS}

\section{General information}

All the experimental rabbits were in good health, showing no abnormalities in emotional state, behavioral activity, coat color or feces. There were no significant differences between the food consumption. There was also no difference in the increasing rate of body weight among the groups.

\section{Effects of puerarin on serum lipids}

The differences in blood lipid levels in rabbits were not significant between groups before the experiment $(P>0.05)$. There was no significant change in lipid levels of the normal group during the whole experiment. On the $30^{\text {th }}$ day after the test was conducted, the levels of TC, TG, LDL-C and AI in the groups fed high-fat diet were significantly higher than those in the normal group $(P<0.05)$, while those in all the medication groups were significantly lower compared with the model group $(P<0.05)$ (except the low-dose puerarin group), and no significant difference was found in HDL-C. The TC and LDL-C levels continued to rise in the model group and treatment group 60 days later, but the levels of TC, LDL-C and AI in each medication group were significantly lower than those in the model group $(P<0.05)$ (except the low-dose puerarin group). On the $90^{\text {th }}$ day, the levels of TC, TG, LDL-C and $\mathrm{AI}$ were significantly lower in the high-dose puerarin group, middle-dose puerarin group and simvastatin group than the model group $(P<0.05)$, and the HDL-C level showed a trend of higher than that in the model group, as shown in Table 1.

\section{Effects of puerarin on blood rheology}

The whole blood hemorheology analysis was conducted on the rabbits in a timely manner after the cardiac blood collection. The results showed that the whole blood viscosity generally increased in the high-fat diet groups, while the whole blood viscosity and plasma viscosity in the medication groups (except the low-dose puerarin group) were significantly lower than those in the model group $(P<0.05)$. There was no significant difference in
Table 3. Aortic staining results $(\mathrm{x} \pm \mathrm{s})$.

\begin{tabular}{cccc}
\hline Group & $\mathrm{n}$ & $\begin{array}{c}\text { Dose } \\
(\mathrm{mg} / \mathrm{kg})\end{array}$ & $\begin{array}{c}\text { Plaque area/ } \\
\text { total area }(\%)\end{array}$ \\
\hline 1 & 10 & & 0 \\
2 & 10 & & $51.33 \pm 12.54$ \\
3 & 10 & 5 & $25.26 \pm 11.42^{*}$ \\
4 & 10 & 400 & $18.24 \pm 7.05^{*}$ \\
5 & 10 & 200 & $26.24 \pm 6.63^{*}$ \\
6 & 10 & 100 & $43.21 \pm 9.77$ \\
\hline
\end{tabular}

Compared with the model group (2), ${ }^{*} P<0.05$.

hematocrit (HCT) and erythrocyte electrophoresis time between the medication groups and the model group. The difference in indicators was not statistically significant in the simvastatin group compared with the model group (Table 2).

\section{Effects of puerarin on pathomorphology}

In the normal group, the arterial wall structures of rabbits were normal from visual inspection, without the formation of atherosclerotic lesion or lipid plaque. In the model group, the aortic intimae of rabbits were rough, and there was a large quantity of visible yellow-white striped fatty streaks and plaques with significant eminence on the intimal surface, in which there were 3 cases with 70\% of aortic intima being atheromatous plaque, occupying almost the full section of arterial intima, while the intimal surfaces of other cases were slightly flat. The intimae in the high-dose puerarin group had no serious lesions generally; one case had smooth intima, without thickening, atherosclerotic lesion or lipid plaque, and the aortic lesion of the other cases was also milder than that in the model group, showing an early phase change of fatty streak. In the middle-dose puerarin group and simvastatin group, although the rabbit aortas showed yellow-white striped fatty streaks and plaques, those of the majority of cases were less compared with the model group, only individual 

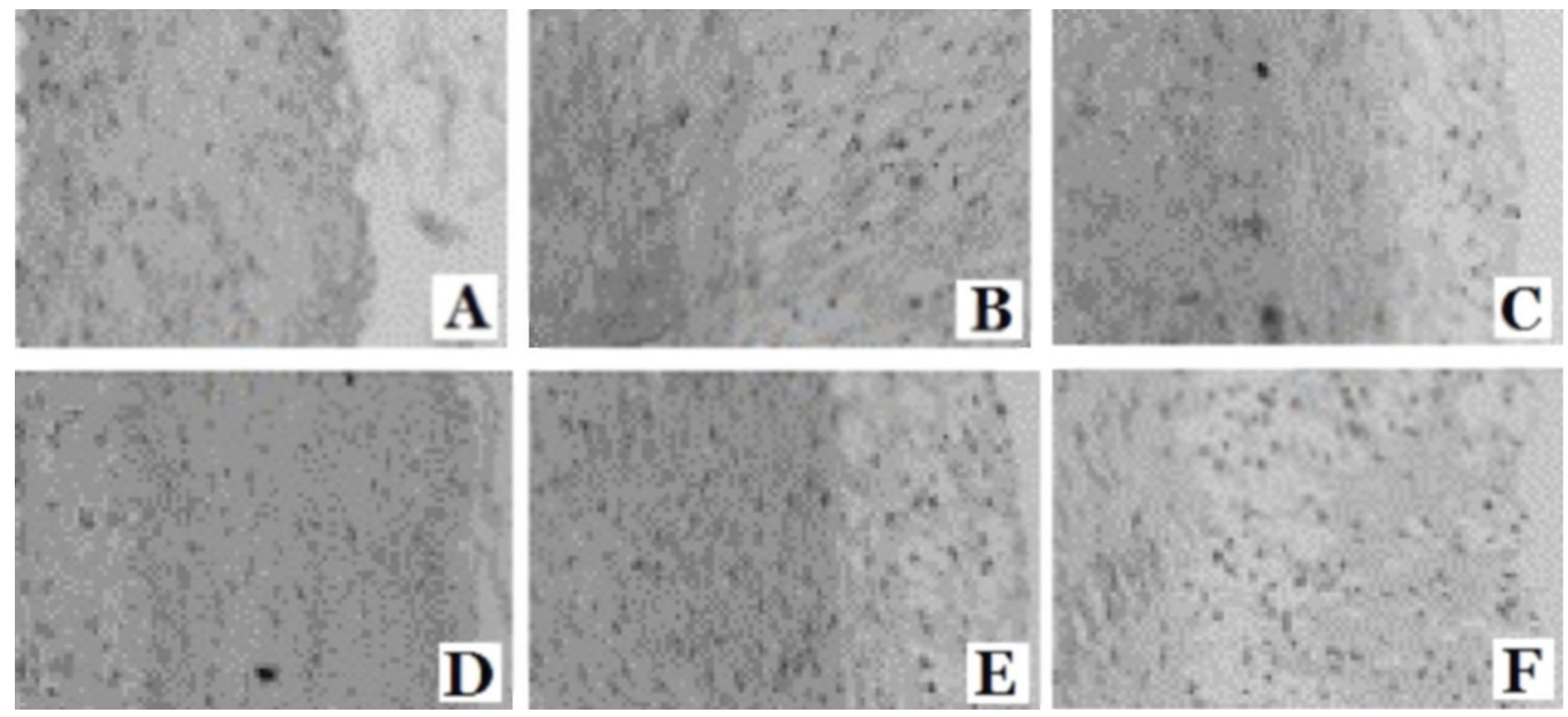

Fig. 1. Light microscopy a ortic observation results. (a) Normal group; (b) model group; (c) simvastatin group; (d) high-dose group; (e) medium-dose group; (f) low-dose group.

Table 4. Effects of puerarin on pathomorphology $(x \pm s)$.

\begin{tabular}{cccccc}
\hline Group & $\mathrm{n}$ & $\begin{array}{c}\text { Dose } \\
(\mathrm{mg} / \mathrm{kg})\end{array}$ & $\begin{array}{c}\mathrm{I} \\
(\mu \mathrm{m})\end{array}$ & $\begin{array}{c}\mathrm{M} \\
(\mu \mathrm{m})\end{array}$ & $\mathrm{I} / \mathrm{M}$ \\
\hline 1 & 10 & & $12.52 \pm 3.24 *$ & $408.95 \pm 42.46$ & $0.03 \pm 0.02 *$ \\
2 & 10 & & $622.35 \pm 23.52$ & $485.64 \pm 47.71$ & $1.29 \pm 0.15$ \\
3 & 10 & 5 & $378.15 \pm 42.16 *$ & $468.19 \pm 45.94$ & $0.82 \pm 0.44 *$ \\
4 & 10 & 400 & $352.74 \pm 45.58 *$ & $438.65 \pm 56.26$ & $0.82 \pm 0.02 *$ \\
5 & 10 & 200 & $447.16 \pm 40.28 *$ & $478.16 \pm 49.57$ & $0.92 \pm 0.33 *$ \\
6 & 10 & 100 & $576.49 \pm 47.55$ & $468.16 \pm 33.46$ & $1.25 \pm 0.14$ \\
\hline
\end{tabular}

Table 5. Aortic PCNA and PDGF-A protein expression levels.

\begin{tabular}{lll}
\hline Group & PCNA & PDGF-A \\
\hline Normal & $0.64 \pm 0.11 * \boldsymbol{\Delta}$ & $0.58 \pm 0.12 * \boldsymbol{\Delta}$ \\
Model & $1.15 \pm 0.05 \# \boldsymbol{\Delta}$ & $1.21 \pm 0.13 \# \boldsymbol{\Delta}$ \\
Simvastatin & $0.76 \pm 0.06 \# *$ & $0.85 \pm 0.07 \# *$ \\
Puerarin & $0.83 \pm 0.12 \# *$ & $0.82 \pm 0.13 \# *$ \\
\hline
\end{tabular}

Compared with the normal group, $\# P<0.05$; compared with the model group, ${ }^{*} P<0.05$; compared with the simvastatin group, $\mathbf{\Delta} P<0.05$.

cases showing slightly larger lesion range. Most cases in the low-dose puerarin group manifested with endometrial thickening and the formation of atherosclerotic plaque. After Oil Red O staining, the percentage of plaque area in the total aortic area was statistically analysed ${ }^{16}$. There were significant differences between the high-dose puerarin group, middle-dose puerarin group, simvastatin group and the model group $(P<0.05)$, while the difference between the low-dose puerarin group and model group was not significant (Table 3 ).
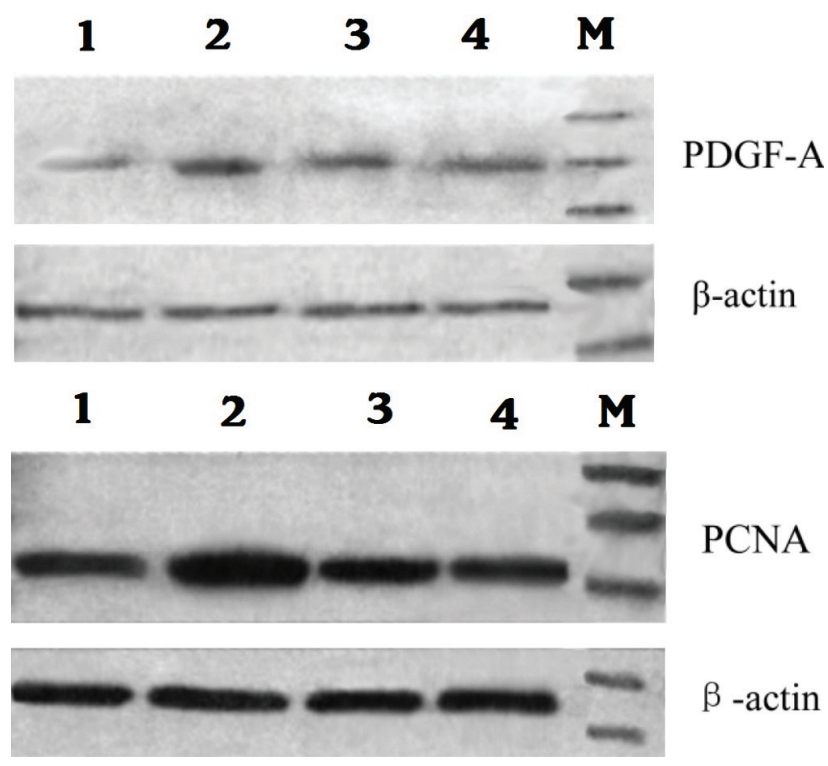

Fig. 2. Western blot results of PCNA and PDGF-A. M: marker; 1: normal group; 2: model group; 3: simvastatin group; 4: highdose puerarin group.

The results (Fig. 1) of aorta observation by light microscopy showed that the aortic structural morphology in the normal group was in a normal state, and the intimal was smooth and complete without any abnormal changes. In the model group, typical AS pathological changes occurred; the number of local smooth muscle cells in the artery wall was reduced; the cells were in a disordered arrangement; pathological changes in the middle- and high-dose puerarin groups were smaller than those in the low-dose puerarin group; the aortic intima 
with pathological changes was slightly thickened; a small amount of foam cells aggregated under endothelial cells; intimal thickening could be seen in the low-dose puerarin group and its foam cell layers were more than those in the middle- and high-lose puerarin groups. In the simvastatin group, some endothelial cells fell off; the structure was relatively complete; the number of foam cell layers was reduced. On each group was conducted wax sealing, slicing and HE staining. The MOTIC AE20 microscope and high-resolution color medical image analysis system were adopted to perform the pathological observation and quantitative analysis of the lesions. Intimal thickness and media thickness were measured and their ratio was calculated for each group with random graphing (Table 4). The results showed that compared with the normal group, I and $\mathrm{I} / \mathrm{M}$ were significantly increased in the model group $(P<0.05)$, while those in the middle- and high-dose groups and simvastatin group were significantly improved compared with the model group $(P<0.05)$, which indicated that the puerarin and simvastatin of the middle dose or above could significantly inhibited the aortic intimal thickening of rabbits and reduced intimal injury.

\section{Aortic PCNA and PDGF-A protein expression levels}

The test results showed that the PCNA and PDGF-A protein expression levels of rabbit aorta were relatively low on average in the normal control group. In contrast, the protein expression levels in the groups fed with highfat diet were significantly increased $(P<0.05)$. However, those in the high-dose puerarin group and simvastatin group were significantly decreased compared with the model group $(P<0.05)$ (Table 5 and Fig. 2).

\section{DISCUSSION}

Lipid metabolism disorder is the most important basis of the pathophysiology of AS. The increase in cholesterol, triglyceride and low-density lipoprotein and the decrease in high-density lipoprotein in blood can promote the deposition of cholesterol in the arterial wall to form AS and cause abnormal hemorheology. It has been confirmed that hyperlipidemia is an important risk factor for CCVD. Therefore, how to effectively control blood lipids and reduce blood specific viscosity is a key to the treatment of ischemic CCVD. Genetic, epidemiological and clinical studies have demonstrated that elevated serum cholesterol is positively correlated with the incidence of coronary heart disease, and to lower serum cholesterol concentration and improve blood flow changes can prevent the occurrence and development of coronary heart disease and other cardio-cerebrovascular diseases ${ }^{17,18}$. Our previous studies showed that puerarin has significant effect as anti-AS, but its mechanism is not clear. In this study, the effect of puerarin on the protein expression of SMC proliferation-related factors was detected from the start with VSMC proliferation in order to further clarify the anti-AS function of puerarin.

The results of this study showed that the levels of serum TC, TG, LDL-C and AI significantly rose 30 days after the rabbits were fed a hyper-lipid diet, and 90 days later, obvious AS plaques occurred but at the same time, giving puerarin of more than the middle dose to the rabbits reduced blood lipid levels and the atherogenic index. The morphological examination found that puerarin significantly reduced the atherosclerotic plaque area, the damage of endothelial cells and elastic plate, the number of foam cells and the proliferation of media vascular SMC. The results of blood rheology analysis showed that puerarin in the middle and high-dose puerarin groups significantly reduced the whole blood viscosity of rabbits after feeding the high-fat diet. There was a significant difference in improving the blood flow change between the two groups and the positive control group, which indicated that both puerarin and simvastatin had a hypolipidemic effect, but the former was better than the latter in improving the blood flow change.

In the pathological process of AS, the proliferating $\mathrm{SMC}$ is one of the main components in plaque, as well as an important factor in determining vascular activity and vascular structure. These changes in vascular structure and function are actually hyperplasia and hypertrophy of VSMC, in which SMC phagocytizing lipid and becoming foam cells is the important part in the formation of AS (ref. ${ }^{19}$ ).

Through establishing the AS rabbit model by feeding high-fat diet, this study showed plaque formation through the general observation on aorta and HE staining by light microscopy, and also demonstrated that the SMC-sourced foam cells existed in plaque. Proliferating cell nuclear antigen (PCNA) is a nuclear protein, and its expression is associated with cell proliferation ${ }^{19}$. The experimental results showed that after the feeding of the high-fat diet, there was large-quantity PCNA expression in the model group, with only a small amount in the normal group, and those in the simvastatin group and puerarin group were also less compared with the model group. The mechanism that media vascular SMC proliferates and directly migrates to intima is very complicated, with many growth factors involved in this process. With the action of growth factors, VSMC is activated, and its phenotype changes, which ultimately leads to endothelial cell damage, while the injured endothelial cells can activate platelets, and variant SMC can release a large amount of PDGF in the form of autocrine and paracrine. PDGF, as an important mitogen, can stimulate the cell division and proliferation of a variety of cells combined with their corresponding membrane PDGF receptors. It is an important factor in inducing SMC proliferation and migration from arterial media to intima. Therefore, inhibiting the migration and proliferation of VSMC by suppressing the PDGF expression is also a new target for treatment of $\mathrm{AS}$ in recent years ${ }^{20-22}$.

This study showed that puerarin inhibited the expression of PCNA and PDGF in rabbits in the pathological process of $\mathrm{AS}$, decrease $\mathrm{SMC}$ proliferation, and significantly reduce the formation of plaque. Therefore, we suggest that puerarin may play a role as anti-AS by reducing the expression levels of PCNA and PDGF and inhibiting SMC proliferation. In short, the above results suggest that 
puerarin has a significant effect on anti-AS, and regulates blood lipids and improving blood rheology may be one of its mechanisms of action.

\section{ACKNOWLEDGEMENT}

This research is funded by the following projects:the Nature Science Foundation of Inner Mongolia Autonomous Region (2013MS1224).

Authorship contributions: LB: literature search; YW, $\mathrm{AB}$ : manuscript writing; RM: study design; $\mathrm{RC}$ : data collection; XR: data analysis; YZ: data interpretation; GW: statistical analysis, figures.

Conflict of interest statement: None declared.

\section{REFERENCES}

1. LaRosa JC, Grundy SM, Waters DD, Shear C, Barter P, Fruchart JC, Gotto AM, Greten H, Kastelein JJ, Shepherd J, Wenger NK. Intensive Lipid Lowering with Atcrvastatin in Patients with stable coronary disease. N Eng J Med 2005;352:1425-35.

2. Pan D, Yang J, Lu F, Xu D, Zhou L, Shi A, Cao K. Platelet- derived growth factor BB modulates PCNA protein synthesis partially through the transforming growth factor beta signaling pathway in vascular smooth muscle cells. Biochem Cell Biol 2007;85:606-15.

3. Keramati AR, Singh R, Lin A, Faramarzi S, Ye ZJ, Mane S, Tellides G, Lifton RP, Mani A. Wild-type LRP6 inhibits, whereas atherosclerosislinked LRP6R611C increases PDGF-dependent vascular smooth muscle cell proliferation. Proc Natl Acad Sci U S A 2011;108:1914-8.

4. Qi H, Li L, Huang C, Li W, Wu C. Optimization and physicochemical characterization of thermosensitive poloxamer gel containing puerarin for ophthalmic use. Chem Pharm Bull (Tokyo) 2006;54:1500-7.

5. Zhu YM, Ni C, Zhu L, Shen YL, Chen YY. [Effect and mechanism of puerarin on high glucose-induced hypo-responses in vascular contraction]. Zhongguo Ying Yong Sheng Li Xue Za Zhi 2011;27:62-5.

6. Wang $Q, X u X$. [Progresses in research of hemolysis induced by puerarin injection]. Zhongguo Zhong Yao Za Zhi 2011;36:1402-5.

7. Tian F, Xu LH, Zhao W, Tian LJ, Ji XL. The optimal therapeutic timing and mechanism of puerarin treatment of spinal cord ischemiareperfusion injury in rats. J Ethnopharmacol 2011;134:892-6.

8. Li DZ, Hu YF, Yang KP. [Protective effect of puerarin on endothelia dysfunction of heat shock protein 60 induced specific immunity in apolipoprotein E-null mice]. Zhongguo Zhong Xi Yi Jie He Za Zhi 2006;26 Suppl:4-6.
9. Chen R, Xue J, Xie M. Puerarin prevents isoprenaline-induced myocardial fibrosis in mice by reduction of myocardial TGF-beta1 expression. J Nutr Biochem 2012;23:1080-5.

10. Liu JM, Ma L, He WP. [Therapeutic effect of puerarin therapy on sudden deafness]. [Clinical Trial Comparative Study Randomized Controlled Trial]. Di Yi Jun Yi Da Xue Xue Bao 2002;22:1044-5.

11. Liu R, Xing D, Lu H, Wu H, Du L. Pharmacokinetics of puerarin and ginsenoside $\mathrm{Rg} 1$ of $\mathrm{CBN}$ injection and the relation with platelet aggregation in rats. Am J Chin Med 2006;34:1037-45.

12. Zhang S, Ji G, Liu J. Reversal of chemical-induced liver fibrosis in Wistar rats by puerarin. J Nutr Biochem 2006;17:485-91.

13. Nakamura K, Nishihata $T$, Jin JS, Ma CM, Komatsu K, Iwashima M, Hattori M. The C-glucosyl bond of puerarin was cleaved hydrolytically by a human intestinal bacterium strain PUE to yield its aglycone daidzein and an intact glucose. Chem Pharm Bull (Tokyo) 2011;59:23-7.

14. Lv Y, Hughes TC, Hao X, Mei D, Tan T. Preparation of monomeric and polymeric beta-cyclodextrin functionalized monoliths for rapid isolation and purification of puerarin from Radix puerariae. J Sep Sci 2011; doi: 10.1002/jssc.201100282

15. Luo CF, Yuan M, Chen MS, Liu SM, Zhu L, Huang BY, Liu XW, Xiong W. Pharmacokinetics, tissue distribution and relative bioavailability of puerarin solid lipid nanoparticles following oral administration. Int J Pharm 2011;410:138-44.

16. Dong $P, M a C J$, Wendusu B, Wang B, Han XM, Wang Y, Song MD, Du GE. [Effects of Eerdun-wurile in a Rabbit Model of Atherosclerosis]. Chinese Archives of Traditional Chinese Medicine 2011;28:1012-5.

17. Tavridou A, Efthimiadis A, Efthimiadis I, Manolopoulos VG Simvastatin-induced changes in circulating oxidized low-density lipoprotein in different types of dyslipidemia. Heart Vessels 2010;25:288-93.

18. Feaver RE, Gelfand BD, Wang C, Schwartz MA, Blackman BR Atheroprone hemodynamic regulates fibronectin deposition to create positive feedback that sustains endothelial inflammation. Circ Res 2010;106:1703-11.

19. Dzau VJ, Braun-Dullaeus RC, Sedding DG. Vascular Proliferation and Atherosclerosis: New Perspectives and Therapeutic Strategies. Nat Med 2002;8:1249-56.

20. Kappert K, Sparwel J, Sandin A, Seiler A, Siebolts U, Leppänen O, Rosenkranz S, Ostman A. Cultured VSMCs and in Restenosis Antioxidants Relieve Phosphates Inhibition and Reduce PDGF Signaling in Cultured VSMCs and in Restenosis. Arterioscler Thromb Vasc Biol 2006;26:2644-51.

21. Brown XQ, Bartolak-Suki E, Williams C, Walker ML, Weaver VM, Wong JY. Effect of substrate stiffness and PDGF on the behavior of vascular smooth muscle cells: implications for atherosclerosis. J. Cell Physiol 2010;225:115-22.

22. Li L, Blumenthal DK, Terry CM, He Y, Carlson ML, Cheung AK. PDGFInduced Proliferation in Human Arterial and Venous Smooth Muscle Cells: Molecular Basis for Differential Effects of PDGF Isoforms. J Cell Biochem 2011;112:289-98. 